\title{
New schemes of compact modal LC modulators for control of light spatial structure
}

\author{
Svetlana Kotova*, Alexandra Mayorova, and Sergey Samagin \\ Lebedev Physical Institute RAS, Samara branch, 221, Novo-Sadovaya Str., Samara, 443011 Russia
}

\begin{abstract}
The results of numerical simulation of new schemes of compact modal LC spatial modulators are presented. Special configurations of contact electrodes and predetermined values of applied voltages provide the jump in the profile of phase delay of light passing through the modulator or reflecting from it. This leads to the appearance of a vortex field. Ring-shaped light fields and light fields with intensity distribution in the form of two maxima, which rotate in the transverse plane during propagation were obtained. These devices may be valuable for use in the spectroscopy of single molecules.
\end{abstract}

Structured light has a huge number of applications, including microscopy and spectroscopy [1]. Formation of light fields with various (arbitrary) intensity distributions and dynamic control of their parameters is possible using multi-pixel LC SLM. For practical applications the reducing cost of modulators as well as the simplification and reducing the sizes of the optical schemes on their base are of interest. In this paper we present new schemes of compact modal LC light modulators which can form certain types of vortex light fields.

The modal principle of control has been proposed in studies $[2,3]$. The important feature of those modulators is the presence of additional homogeneous transparent high-resistance layer. This layer covers the low-resistance coating with the hole and performs the function of a control electrode. Tunable spherical and cylindrical lenses were designed in this way. Next, we developed LC focusator (or 4-channel LC modulator) [4, 5]. By using LC focusator as an optical transparent it is possible to focus light into a point spot or a line segment and to form transverse distributions of intensity. Shaped beams can be of various forms, with sizes, position and orientation controlled by means of applied voltages [6 - 8]. Since only a few control electrodes and low control voltages are used in modal LC devices they are easy to control, have relatively low cost and are easy to manufacture.

To obtain light fields with angular momentum, it is necessary to provide a jump in the phase profile of the light passing through the device. This can be done with the special geometry of electrodes. It is found that axially symmetric light intensity distributions can be obtained for two electrode configurations. In the first case, the contact electrode on one of substrates is divided in half, and in the second one, two narrow stripe electrodes are applied on one of the substrates. Thus, a spiral focusator and spiral phase plate can be realized. Selecting the amplitudes of the potentials in such a way that the magnitude of the jump in the

* Corresponding author: kotova@ fian.smr.ru 
phase profile varies by a factor of $2 \pi$, it is possible to control the diameter of the ring (Fig. 1). It is also possible to move the light ring in the observation plane. This case may be of interest for example for STED-nanolithography.
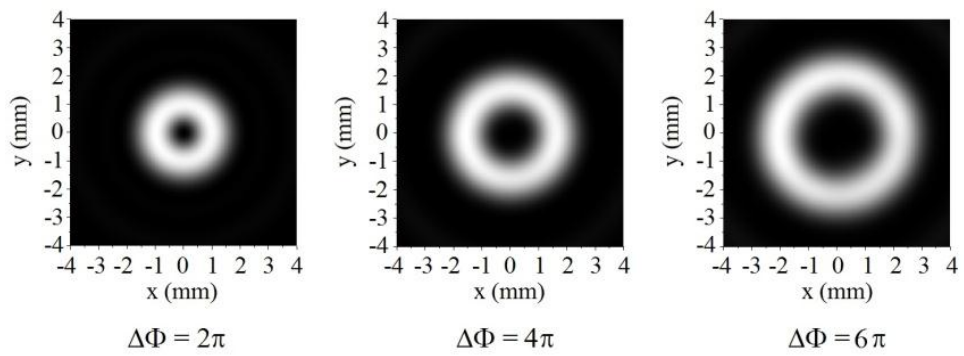

Fig. 1. Dynamic control of the formed fields. Distributions of the intensity in the observation plane for different potentials producing the phase jump $\Delta \Phi$.

If the conductive coating and contact strip electrodes on one of the substrates are divided in the middle by a narrow nonconducting transparent strip, then on this substrate four contact electrodes are formed. With the help of such a device, one can obtain an intensity distribution with two maxima that rotate during propagation. Incident light of different curvatures will produce maxima at different positions in the set observation plane (Fig. 2). This configuration of the modulator can be used in fluorescent nanoscopy [9].

$-0.7 \mathrm{D}$

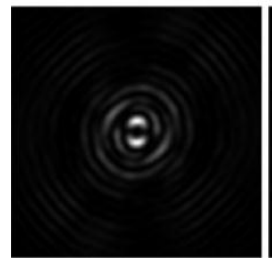

$-0.3 \mathrm{D}$

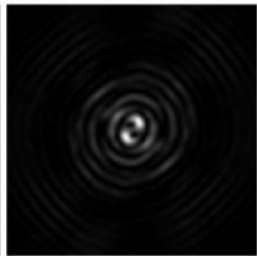

$0 \mathrm{D}$

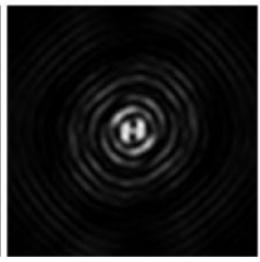

$0.3 \mathrm{D}$

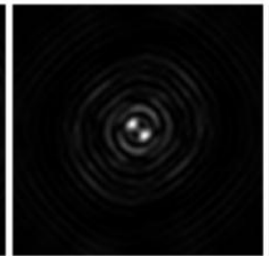

$0.7 \mathrm{D}$

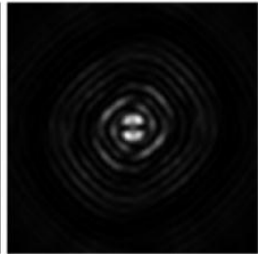

Fig. 2. Intensity distribution of the light in the observation plane for different curvatures (in dioptres) of the incident wave front.

This research is financially supported by the RFBR (projects Nos. 16-02-01055, 16-29-11809 and No. 16-42-630773).

\section{References}

1. H. Rubinsztein-Dunlop et al., Journal of Optics, 19, 013001 (2017)

2. A.F. Naumov, Proc. P.N. Lebedev Physical Institute 217, 182 (1993)

3. A.F. Naumov, M.Y. Loktev, I.R. Guralnik and G, Vdovin, Opt. Lett., 23, 992 (1998)

4. S.P. Kotova, V.V. Patlan and S.A. Samagin, Quantum Electronics, 41, 58 (2011)

5. S.P. Kotova, V.V. Patlan and S.A. Samagin, Quantum Electronics, 41, 65 (2011)

6. A.V. Korobtsov, S.P. Kotova, N.N. Losevsky, A.M. Mayorova and S.A. Samagin, Quantum Electronics, 44, 1157 (2014)

7. S.P. Kotova, A.M. Mayorova and S.A. Samagin, Journal of Optics 17, 055602 (2015)

8. S.P. Kotova, A.M. Mayorova and S.A. Samagin, Journal of Opt. Tech., 84, 323 (2017)

9. A.V. Naumov, Phys. Usp., 56, 605 (2013) 